\title{
An Unusual Case of Bilateral Submandibular Sialolithiasis
}

\author{
Jin Pyeong Kimª, Jung Je Park ${ }^{\mathrm{a}}$, Hee Young Son ${ }^{\mathrm{b}}$, Seung Hoon Woo ${ }^{\mathrm{a}, \mathrm{c}, \mathrm{d}}$
}

\begin{abstract}
Salivary calculi in the pediatric population comprise only $3 \%$ of all cases of sialolithiasis. In addition, the presentation of bilateral calculi in children is rare. Traditionally, the treatment for submandibular calculi in the proximal duct has been a sialadenectomy in spite of its risk.

Recently, minimally invasive techniques have been reported, the Intraoral removal of calculi is useful for preservation of the submandibular gland and preserved gland can restore gland function to normal after stone removal. We would report the 15 -year-old boy case with bilateral submandibular gland hilum sialolithiasis and the intraoral removal only stones.
\end{abstract}

Keywords: Submandibular duct; Salivary gland; Sialolithiasis

Manuscript accepted for publication December 7, 2011

\footnotetext{
a Department of Otolaryngology, Gyeongsang National University, Jinju, Korea

${ }^{\mathrm{b}}$ Department of Otolaryngology, Thyroid/head\&neck cancer center of The Dongnam institute of Radiological \& Medical Sciences (DI RAMS), Pusan, Korea

${ }^{\mathrm{C}}$ Institute of Health Sciences, Gyeongsang National University, Jinju, Korea

${ }^{\mathrm{d}}$ Corresponding author: Seung Hoon Woo, Department of Otorhinolaryngology, Gyeongsang National University, 90 Chilam-dong, Jinju, South Korea, 660-702, Korea.

Email: lesaby@hanmail.net
}

doi:10.4021/jmc466w

\section{Introduction}

While the incidence of sialolithiasis peaks in middleaged adults, it is a rare condition among children [1-4]. Furthermore, bilateral sialolithiasis in the pediatric population is extremely rare. A review of all reports of sialolithiasis in children from 1913 to 2011, never reported bilateral hilum sialolithiasis in all salivary stones.

A trial of conservative therapy usually precedes surgical intervention. In spite of conservative treatments, chronic sialoadenitis of the submandibular gland associated with salivary stones recurs frequently and requires the operative removal in many cases.

We describe a 15 -year-old boy with the sialolithiasis located in the both hilum of submandibular gland removed by intraoral approach. The submandibular gland was preserved, only salivary stones were removed and review of the literature.

\section{Case Report}

A 15 year-old-boy was admitted for the chief complaint of the postprandial swelling of the both submadibular area of neck. This symptom was initiated 2 to 3 years ago. In physical examination, a diffuse swelling was observed in the both submandibular area, slight tenderness was associated, but significantly palpable lymph nodes were not detected in the neck. Under the suspicion of submandibular duct stones, neck computed tomography was performed. The result showed stones smaller than $1 \mathrm{~cm}$ in the hilum of both submandibular gland, and tiny stones in the parenchyma of submandibular gland (Fig. 1). The patient was so young and his salivary secretion would be markedly decreased. For that reason, if the bilateral submandibular gland was resected, this could cause a discomfort in his whole life. So we planned to remove the stones by intraoral approach to preserve the submandibular gland.

The mouth floor was exposed sufficiently and oral mucosa on the mouth floor. After the careful assessment of the lingual nerve and the wharton's duct in the secure operation 


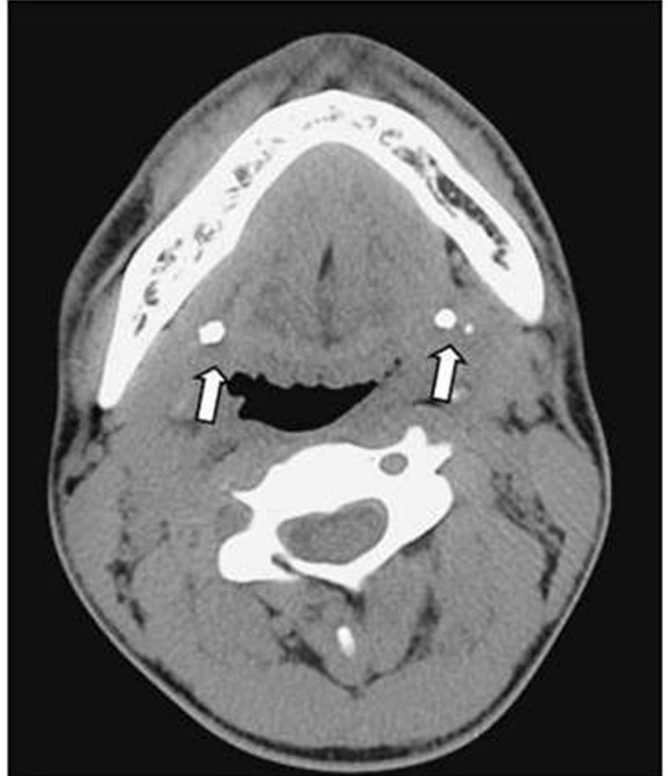

Figure 1. Axial computed tomography scans demonstrating bilateral calcified densities in the submandibular gland (arrows). field. The stone was removed through the stone size incision made on the upper area of the Wharton's duct where the stone was palpated (Fig. 2). Afterward, the occlusion of the incised salivary duct as the original shape was performed, and the contralateral stone was removed by the same method (Fig. 3). Then, there was no complication and the patient is under the follow-up observation at our outpatient clinic now.

\section{Discussion}

Though incidence of sialolithiasis peaks in the third to sixth decades of life, only $3 \%$ of all cases, occur in children and sialolithiasis remains a rare cause of salivary dysfunction in pediatric populations [1-4].

The treatment methods for submandibualar duct stones are conservative care, operative removal, the minimal invasive surgery such as extracorporeal shock wave lithotripsy, sialendoscopy etc. The conservative care method is to administer antibiotics and anti-inflammatory agents and to wait for the spontaneous came out through the orifice of the duct. The method could be applied to the temporary occlusion

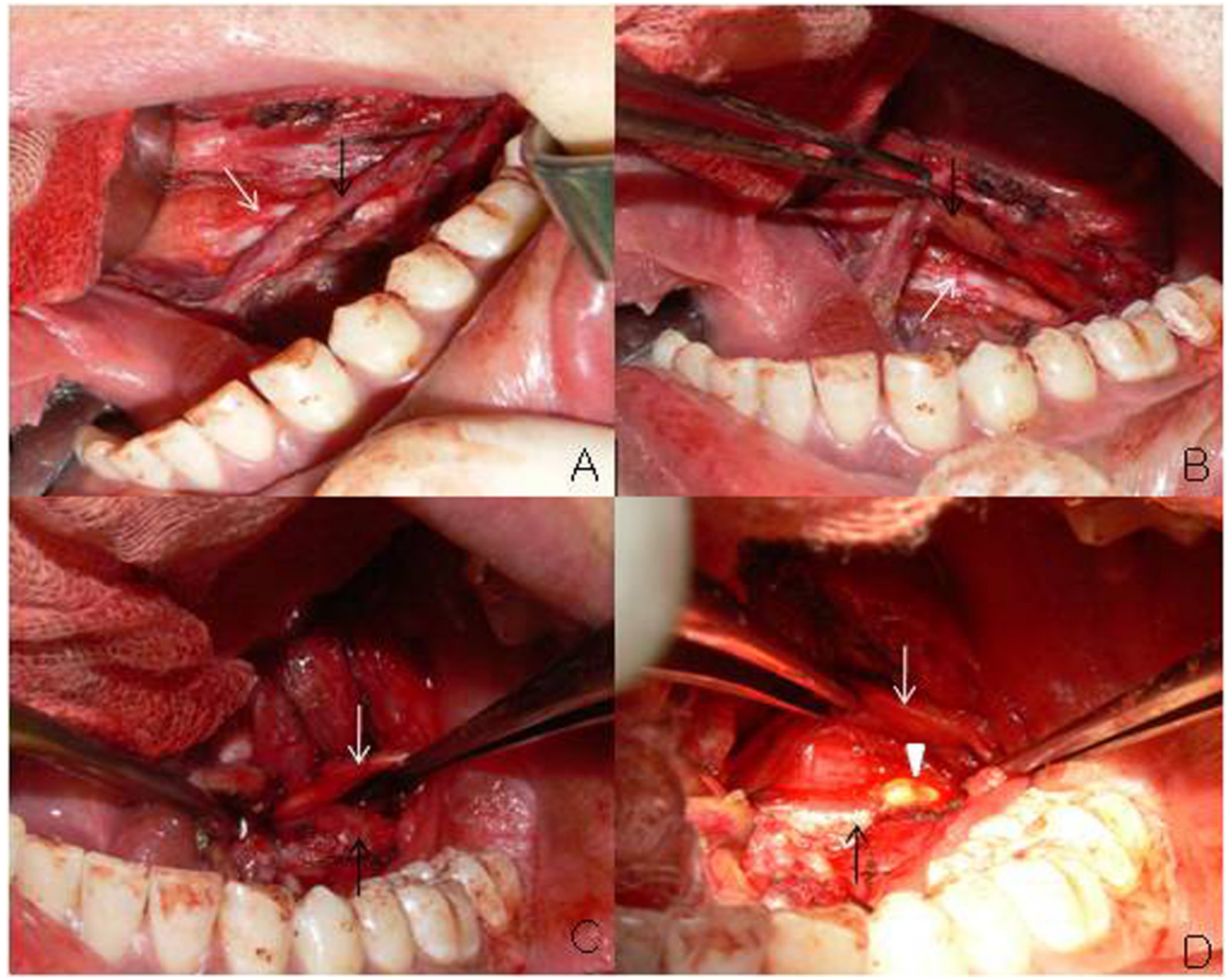

Figure 2. Surgical approach to the stones in the warton's duct or the hilum of the gland. A portion of sublingual gland overlying the proximal duct was removed to expose the proximal duct(black arrow) and lingual nerve(white arrow). The lingual nerve crossing the wharton's duct was retracted to the inside (A,B). The stone in the hilum of submandibular gland was exposed(arrow head) by pushing upward the subman $\neg$ dibular triangle using the fingers and incision made on the upper area of the Wharton's duct where the stone was palpated $(C, D)$. 


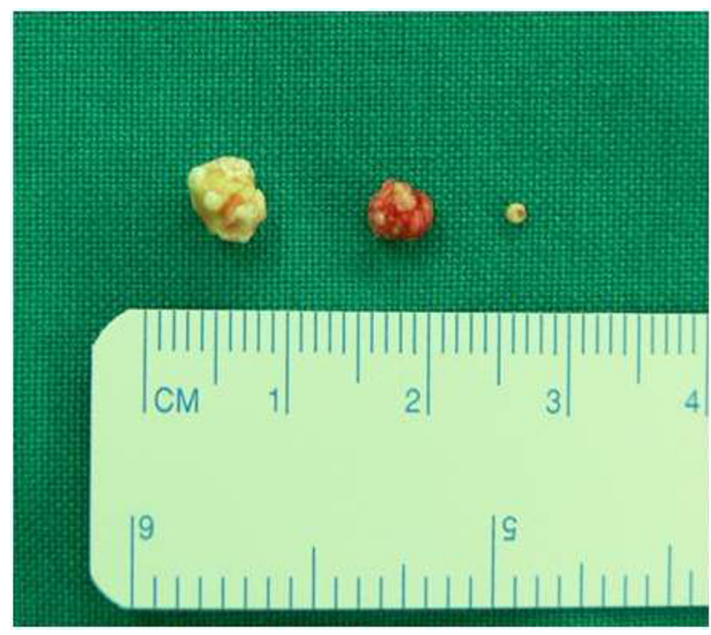

Figure 3. Two stones smaller than $1 \mathrm{~cm}$ in the hilum of both submandibular gland, and tiny one stone in the parenchyma of submandibular gland were removed.

caused by tiny stones within the duct or inflammation. The treatment outcome of extracorporeal shock wave lithotripsy for submandibular duct stones that account for $80-90 \%$ of sialolithiasis has the tendency to be lower than for the parotid stone [4]. Sialendoscopy has advantages that it could diagnose other causatives of the obstruction of Wharton's duct such as stricture, torsion, and polyp and treat them, nonetheless, it has limitations for the cases with large size stones or the cases associated with acute sialoadenitis [5].

For the cases with submandibular duct stones located in the distal area of the Wharton's duct, simple removal of stone has been performed. On the contrary to this, the sialolithiasis in the proximal of the Wharton's duct or sialolithiasis in the parenchyma of the submandibular gland, the excision of submandibular gland has been performed. In the excision of submandibular gland cases, injury of the marginal branch of facial nerve may occur in $12.5 \%$ cases overall, and permanent injury may be also developed in 7\% [6]. In addition, not only the problems of the formation of scar, alteration of dermal sensation, taste formation, also functional prolems such as the reduction of salivation. Because of such problems, recently, in the treatment of submandibular duct stones, efforts have been made to preserve the submandibular gland

According to numerous reports, even if only the submandibular gland of one side was removed, the salivation within the oral cavity was reduced and thus oral hygiene may be deteriorated [5]. In addition, even in the cases determined to require the excision of submandibular gland because chronic inflammation and atrophy of the submandibular gland were suspected to be caused by the stones, in postsurgical histopathological findings, the cases with normal or almost normal excised submandibular gland tissues reached 57\% [7]. According to reported studies, in the patients underwent the transoral removal of submandibular stones, function of the submandibular gland was assessed by scintigraph, and it was found that after the removal of stones, $75 \%$ submandibular glands recovered normal functions $[8,9]$. Therefore, numerous attempts have been made to preserve the submandibular gland. Furthermore, as in our case, in children developed submandibular duct stones bilaterally, more and more the method preserving the submandibular gland should be considered.

In conclusion, our case was a young patient with the stones in the hilum of submandibular gland bilaterally. The removal of stones was performed by the intraoral removal of submandibular stones while preserving both salivary glands.

\section{Grant Support}

No financial or material support has been received for this work; moreover, the authors declare no financial interests in companies or other entities that could have an interest in the information within this contribution.

\section{Conflict of Interest}

None of the authors has any conflict of interest, financial or otherwise.

\section{References}

1. Cummings iCWC, J.M. Fredrickson, L.A. Harker, et al. Otolaryngology Head and Neck Surgery. 1998.

2. Lustmann J, Regev E, Melamed Y. Sialolithiasis. A survey on 245 patients and a review of the literature. Int J Oral Maxillofac Surg. 1990;19(3):135-138.

3. Williams MF. Sialolithiasis. Otolaryngol Clin North Am. 1999;32(5):819-834.

4. Zenk J, Constantinidis J, Al-Kadah B, Iro H. Transoral removal of submandibular stones. Arch Otolaryngol Head Neck Surg. 2001;127(4):432-436.

5. Katz P, Fritsch MH. Salivary stones: innovative techniques in diagnosis and treatment. Curr Opin Otolaryngol Head Neck Surg. 2003;11(3):173-178.

6. Hald J, Andreassen UK. Submandibular gland excision: short- and long-term complications. ORL J Otorhinolaryngol Relat Spec. 1994;56(2):87-91.

7. Kim JY WS, Lee JW, Lee IW, Seo CJ, Lee BJ. Histopathologic changes in submandibular sialolithiasis. Korean J Otolaryngol 2003.

8. Cunning DM, Lipke N, Wax MK. Significance of unilateral submandibular gland excision on salivary flow in noncancer patients. Laryngoscope. 1998;108(6):812815 . 
9. Woo SH, Jang JY, Park GY, Jeong HS. Long-term outcomes of intraoral submandibular stone removal in children as compared with adults. Laryngoscope. 2009;119(1):116-120. 